\title{
Identification of a five-gene signature in association with overall survival for hepatocellular carcinoma
}

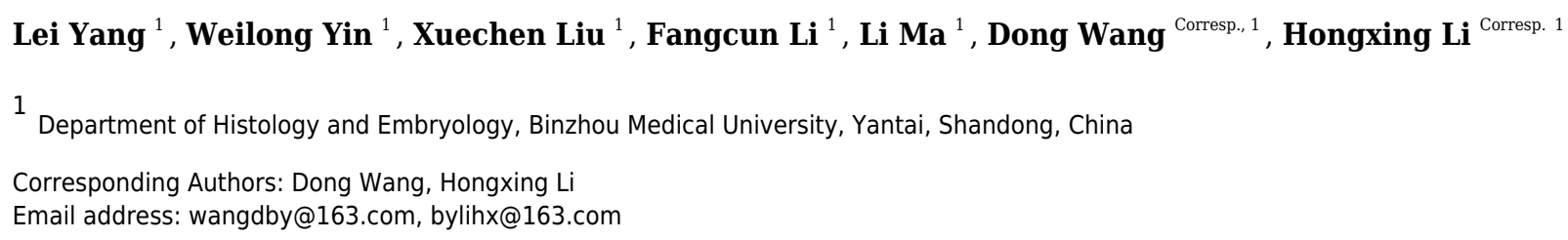

Background. Hepatocellular carcinoma (HCC) is considered to be a malignant tumor with a high incidence and a high mortality. Accurate prognostic models are urgently needed. The present study was aimed at screening the critical genes for prognosis of HCC. Methods. GSE25097, GSE14520, GSE36376 and GSE76427 datasets were obtained from Gene Expression Omnibus (GEO). We used GEO2R to screen differentially expressed genes (DEGs). A protein-protein interaction network of the DEGs was constructed by Cytoscape in order to find hub genes by module analysis. The Metascape was performed to discover biological functions and pathway enrichment of DEGs. MCODE components were calculated to construct a module complex of DEGs. Then, gene set enrichment analysis (GSEA) was used for gene enrichment analysis. ONCOMINE was employed to assess the mRNA expression levels of key genes in HCC. And the survival analysis was conducted using the array from The Cancer Genome Atlas (TCGA) of HCC. Then, performing the LASSO Cox regression model to establish and identify the prognostic gene signature. And we validated the prognostic value of the gene signature in TCGA cohort. Results. We screened out 10 hub genes which were all up-regulation in HCC tissue. They mainly enrich in mitotic cell cycle process. The GSEA results showed that these data sets had good enrichment score and significance in the cell cycle pathway. Each candidate gene may be an indicator of prognostic factors in the development of HCC. However, hub genes expression was weekly associated with overall survival in HCC patients. LASSO Cox regression analysis validated a five-gene signature (including CDC20, CCNB2, NCAPG, ASPM and NUSAP1). These results suggest that five-gene signature model may provide clues for clinical prognostic biomarker of HCC. 


\section{Identification of a five-gene signature in association} 2 with overall survival for hepatocellular carcinoma

3

4

5 6

7 8 9

\author{
Lei Yang ${ }^{1}$, Weilong Yin ${ }^{1}$, Xuechen Liu ${ }^{1}$, Fangcun $\mathrm{Li}^{1}, \mathrm{Li} \mathrm{Ma}^{1}$, Dong Wang ${ }^{1 *}$, Hongxing Li ${ }^{1 *}$ \\ ${ }^{1}$ Department of Histology and Embryology, Binzhou Medical University, Yantai 264003, \\ Shandong, P. R. China \\ Corresponding Author: \\ Dong Wang ${ }^{1}$ \\ Department of Histology and Embryology, Binzhou Medical University, \#346 Guanhai Road, \\ Yantai, Shandong, 264003, P. R. China. \\ Email address: wangdby@163.com \\ Hongxing $\mathrm{Li}^{1}$ \\ Department of Histology and Embryology, Binzhou Medical University, \#346 Guanhai Road, \\ Yantai, Shandong, 264003, P. R. China. \\ Email address: bylihx@163.com
}




\section{Abstract}

22

Background. Hepatocellular carcinoma (HCC) is considered to be a malignant tumor with a high incidence and a high mortality. Accurate prognostic models are urgently needed. The present study was aimed at screening the critical genes for prognosis of HCC. Methods. GSE25097, GSE14520, GSE36376 and GSE76427 datasets were obtained from Gene Expression Omnibus (GEO). We used GEO2R to screen differentially expressed genes (DEGs). A protein-protein interaction network of the DEGs was constructed by Cytoscape in order to find hub genes by module analysis. The Metascape was performed to discover biological functions and pathway enrichment of DEGs. MCODE components were calculated to construct a module complex of DEGs. Then, gene set enrichment analysis (GSEA) was used for gene enrichment analysis. ONCOMINE was employed to assess the mRNA expression levels of key genes in HCC. And the survival analysis was conducted using the array from The Cancer Genome Atlas (TCGA) of HCC. Then, performing the LASSO Cox regression model to establish and identify the prognostic gene signature. And we validated the prognostic value of the gene signature in TCGA cohort. Results. We screened out 10 hub genes which were all up-regulation in HCC tissue. They mainly enrich in mitotic cell cycle process. The GSEA results showed that these data sets had good enrichment score and significance in the cell cycle pathway. Each candidate gene may be an indicator of prognostic factors in the development of HCC. However, hub genes expression was weekly associated with overall survival in HCC patients. LASSO Cox regression analysis validated a five-gene signature (including CDC20, CCNB2, NCAPG, ASPM and NUSAP1). These results suggest that five-gene signature model may provide clues for clinical prognostic biomarker of HCC.

\section{Introduction}

The incidence rate of hepatocellular carcinoma (HCC) ranks sixth among all malignant tumors and the mortality rate ranks third (1). More than 580,000 new cases are expected in Asia every year (2). The genetic aberrations, cellular environment and environmental effects are considered as responsible for the development, progression and metastasis of HCC (1).

Genomic research has been the focus of hepatocellular carcinoma treatment (3). Recently, highthroughput platform microarrays for analyzing gene expression have been widely developed (4) as an effective tool for identifying general genetic changes during tumorigenesis (5). Microarray techniques can not only find related genes of diseases, targets of anti-tumor drugs, but also prognostic analysis of tumor patients, and can reveal the relationship between gene expression and regulation. In clinical research, it also plays the role of providing ideas for the diagnosis and treatment of certain diseases (6). We found that there have been studies exploring the prognostic signatures of colon cancer and lung adenocarcinoma, but the prognostic signatures of hepatocellular carcinoma need to be supplemented $(7,8)$. In this study, we chose four GEO series (GSE25097, GSE14520, GSE36376 and GSE76427) including hepatocellular carcinoma tumor tissue and non-tumor tissue samples. We use GEO2R to screen out differentially expressed genes (DEGs) and to find hub genes by constructing their 
60 protein interaction network. This study is aimed at validating some potential targets to effectively

61 assist clinical workers to predict overall survival of HCC patients.

\section{Materials \& Methods}

63 Data adoption criteria

64 We have looked for publicly available series from the GEO Repository browser. Using

65 "hepatocellular carcinoma" as a keyword, a total of 450 series were retrieved. Sort these series

66 acting in accordance with the number of samples, set study type to "Expression profiling by

67 array" and organism to "Homo sapiens", and look for the series with normal tissue and

68 hepatocellular carcinoma tissue control. Make sure that the normal samples are taken from

69 adjacent tissues of HCC patients. In the end, four HCC gene expression profiles (GSE14520,

70 GSE25097, GSE36376 and GSE76427) were selected because they have more and better-quality

71 samples. The normal samples in these four series were all taken from adjacent tissues of HCC

72 patients. Data were downloaded from the publicly available database hence it was not applicable

73 for additional ethical approval.

74 DEGs analysis

75 GEO2R (http://www.ncbi.nlm.nih.gov/geo/geo2r/) was implemented to screen out DEGs

76 between HCC tumor and non-tumor tissue samples. After we obtain the data, we used Bio Tools

77 v5.0 (http://www.chrislifescience.club:3838/R/AnnoE2/) to draw a volcano map to find

78 statistically significant differentially expressed genes. The adjusted $\mathrm{P}<0.01$ and $|\operatorname{logFC}| \geq 1$ were

79 set as the threshold, so the false positive result was eliminated as much as possible. In order to

80 eliminate the background error caused by different research units on different platforms, we use

81 the Venny 2.1.0 (https://bioinfogp.cnb.csic.es/tools/venny) mapping to screen out the shared

82 DEGs.

83 PPI network construction

84 We imported initially screened genes into the STRING database (http://string-db.org/), web-

85 based software designed to calculate the integration of protein-protein interactions (9) to obtain

86 the highest confident genes (0.900). Then, the MCC algorithm in Cytohubba which an APP in

87 Cytoscape (Version: 3.7.2) was utilized to screen out the hub genes (10).

88 Functional and pathway enrichment analysis

89 Metascape (http://metascape.org) was performed to analyze process enrichment analysis and

90 pathway analysis of neighbor genes of hub genes. On the basis of Metascape tool, The Gene

91 Ontology (GO) terms, Kyoto Encyclopedia of Genes and Genomes (KEGG) pathways and

92 Reactome Gene Sets can be analyzed. Terms with a $\mathrm{P}$ value $<0.01$, minimum count of 3 , and an

93 enrichment factor $>1.5$ are collected and grouped into clusters depended on their membership

94 similarities. Moreover, the MCODE algorithm of the network is used to identify closely

95 connected neighborhoods of proteins. (11-13).

96 Gene Set Enrichment Analysis (GSEA) analysis

97 GSEA (v4.0.3) was used to verify the results of Metascape analysis $(14,15)$. The gene sets file

98 (C2 KEGG v7.0 symbols), phenotype labels file, and expression dataset file and chip annotation

99 file were prepared and loaded into GSEA. $\mathrm{P}<0.05$ was considered statistically significant. 
100 The hub genes' transcription level analysis in patients with HCC

101

102

103

104

105

106

107

108

109

110

111

112

113

114

115

116

117

118

119

120

121

122

123

124

125

126

127

128

129

130

131

132

133

134

135

136

137

138

139

ONCOMINE (www.oncomine.org) was performed to analyze the mRNA levels of hub genes in

HCC $(16,17)$. Threshold limits were as follows: the data type was mRNA, fold change $=2$ and $P$ $=0.01$. Differential analysis between normal and tumor tissues was performed for each gene. Meanwhile, we compared the level of hub genes expression between normal and tumor tissues by Gene Expression Profiling Interactive Analysis (GEPIA 2) (18). We set $|\log 2 \mathrm{FC}|$ Cutoff to 1, Jitter Size to 0.4, P-value Cutoff to 0.01, and compared HCC tumor samples $(\mathrm{n}=369)$ from the TCGA database with normal samples $(\mathrm{n}=160)$ from the TCGA databases.

Survival analysis of hub genes

The Kaplan-Meier plotter (www.kmplot.com) contains gene expression data from 364 clinical HCC patients derived from TCGA (19). According to the median expression, these samples were divided into a low expression group and a high expression group. The Kaplan-Meier plotters were used to calculate the relapse-free survival (RFS), progression-free survival (PFS) and overall survival (OS) of all liver cancer patient samples.

Establishment of the prognostic gene signature

The mRNA expression and clinical data were downloaded from TCGA-LIHC and cBioportal, including 374 TCGA-LIHC and 50 normal control samples. All patients with a follow-up period less than 60 days were excluded for survival analysis. A prognostic gene signature was constructed based on the results of the least absolute shrinkage and selection operator (LASSO). Cox regression model coefficients $(\beta)$ multiplied with its mRNA expression level. The risk score $=($ Bgene $1 *$ expression level of gene 1$)+($ Bgene $2 *$ expression level of gene 2$)+($ Bgene $3 *$ expression level of gene 3$)+\ldots+($ Bgene $n *$ expression level of gene $n)(20)$. We used the Survminer R package to find the optimal cut-off values. Then the Kaplan-Meier survival curve combined with a log-rank test was performed to compare the difference in overall survival between the high-risk score group and low-risk score group.

Statistical analysis

GraphPad Prism version 8.0 and R software version 4.0.2 (GraphPad Software Inc., USA) was used for statistical analyses. All tests were two-sided, $\mathrm{P}<0.05$ was considered statistically significant.

\section{Results}

Identification of DEGs

We did the research as described in the flow chart (Fig.1). In order to screen the difference of gene expression between HCC and normal liver tissue, four gene expression series (GSE14520, GSE25097, GSE36376 and GSE76427) were downloaded from the GEO database. The profile of GSE14502 includes $225 \mathrm{HCC}$ tumor tissues and 220 non-tumor tissues. The profile of GSE25097 includes $268 \mathrm{HCC}$ tumor tissues and 243 non-tumor tissues. The profile of GSE36376 includes $240 \mathrm{HCC}$ tumor tissues and 193 non-tumor tissues. The profile of GSE76427 includes $115 \mathrm{HCC}$ tumor tissues and 52 non-tumor tissues (Table 1). We found 1095 DEGs in GSE14520 (Fig.2A), 1872 DEGs in GSE25097 (Fig. 2B), 688 DEGs in GSE36376 (Fig. 2C) and 488 DEGs in GSE76427 (Fig. 2D). Among them, 142 DEGs were detected in all

Peer) reviewing PDF | (2020:11:55265:1:1:NEW 11 Feb 2021) 
140 four datasets (Fig. 2E) and all their expressions were matched, including 26 up-regulated genes 141 (Fig. 2F) and 116 down-regulated genes (Fig. 2G) in HCC tumor tissue samples compared with 142 non-tumor liver tissue samples.

143 PPI network construction and co-expression analysis in patients with HCC

144 Next, we imported 142 genes into the STRING webpage software for PPI network construction.

145 After setting the minimum required interaction score to highest confidence (0.900), we obtained

146121 nodes and 398 edges. Then, a cluster network was created by using the MCL cluster

147 algorithm in the STRING website. The first cluster included 16 genes (AURKA, CDKN3, 148 CCNB2, CDC20, PTTG1, MELK, RACGAP1, PRC1, TOP2A, NUSAP1, ASPM, NCAPG, 149 RFC4, PHLDA1, MCM6 and MCM2 (Fig. 3A). Next, we applied Cytohubba's MCC algorithm 150 to rank and obtained the top 10 central genes. They were CDC20, CCNB2, AURKA, ASPM, NCAPG, NUSAP1, CDKN3, PRC1, MELK and TOP2A (Fig. 3B). Interestingly, these ten genes were all within the first cluster created by the MCL clustering algorithm. Functional and pathway enrichment analyses We used the Benjamini and Yekutieli method to adjust the $\mathrm{P}$ value of the enrichment analysis results, and used the standard of adj. $\mathrm{P}<0.05$ to screen for significant enrichment regions. GO terminology enrichment analysis indicated that 10 genes were mainly enriched in cell division, mitotic cell cycle process, DNA conformation change, positive regulation of apoptotic process, DNA-dependent ATPase activity, spindle pole and neural precursor cell proliferation. On the other side, KEGG pathway and Reactome gene sets based analysis revealed that the genes were mainly enriched in the cell cycle (Fig. 4A, B and C). We used PPI network construction in Metascape, and extracted the most important MCODE components from it, and performed functional and pathway enrichment analysis for each MCODE component. The results indicated that the candidate genes of the cell cycle pathway may be indicators of prognostic factors in patients with HCC (Fig. 4D). In order to validate that the cell cycle pathway was related to HCC, we performed GSEA on four databases, GSE25097, GSE14520, GSE36376 and GSE76427. The results showed that these data sets had good enrichment score and significance in the cell cycle pathway (Fig. 4E-4H). The transcription level of Hub genes in HCC In order to further verify whether the differentially expressed genes were overexpressed in HCC patients, the ONCOMINE database was used to compare the expression of those genes in tumor tissues and normal tissues. The analysis results showed that these 10 hub genes are overexpressed in liver cancer (Fig. 5A). Then, we used GEPIA2 to draw Box Plots to visualize the different mRNA expression levels of 10 hub genes between cancer samples and normal samples. The result revealed that the expression levels of these 10 genes in HCC tumor samples were higher than in normal samples (Fig. 5B-5K). Establishment of the five-gene-based prognostic gene signature respectively. The results showed that the OS, PFS and RFS of HCC samples with high expression of those single genes were worse than those with low expression (Fig.6) (Table 2). 
180 Next, the HCC data set of cBioPortal online platform (TCGA, firehose legacy) was applied to

181

182

183

184

185

186

187

188

189

190

191

192

193

194

195

196

197

198

199

200

201

202

203

204

205

206

207

208

209

210

211

212

213

214

215

216

217

218

219

get prognostic information of 10 hub genes. The results point out that there is no significant correlation between the hub genes alteration and decreased OS (Fig.7A). So, we develop a fivegene prognostic signature by LASSO Cox regression analysis. The five genes identified were Cell Division Cycle 20 (CDC20), Non-SMC Condensin I Complex Subunit G (NCAPG), Cyclin B2 (CCNB2), Assembly Factor for Spindle Microtubules (ASPM) and Nucleolus and Spindle Associated Protein 1 (NUSAP1). Then, we validated the prognostic significance of the prognostic signature in $\mathrm{HCC}$ patients by the online cBioPortal platform. The results showed that 5 genes were altered in $93(25 \%)$ of 372 samples (Fig.7C) and those genes alterations were significantly associated with decreased OS (Fig.7B).

We also calculated the five-gene based risk score for each patient from TCGA-LIHC cohort and GSE14520 cohort. The risk score $=0.12 *$ ExpressionCDC20 $+(-0.082) *$ ExpressionCCNB2 $+0.039 *$ ExpressionNCAPG $+0.014 *$ ExpressionASPM $+(-0.04) *$ ExpressionNUSAP1. The Survminer R package was performed to find the optimal cut-off the risk score. Patients in the high-risk score group shown significantly poorer OS than ones in the low-risk score group (from TCGA $p<0.001$ and from GSE14520 p=0.0003) (Fig.7D, E). Our results indicated a good performance of the five-gene signature for survival predication.

\section{Discussion}

The outcome of HCC patients is not only determined by tumor stage, tumor size, serum markers and liver function, but also closely related to some gene's expression in tumor tissue (20). Several researchers have focused on the potential role of gene-signatures based on aberrant mRNA in prognosis prediction of HCC (21-25). In this study, we screened out 10 hub genes by constructing a PPI network and using cytohubba. Enrichment analysis revealed that most of them are involved in cell cycle progression and survival analysis indicates that HCC patients with the abnormal expression of these genes showed poor OS and PFS. We established a 5-gene signature (including CCNB2, CDC20, NUSAP1, ASPM, and NCAPG) for HCC prognosis prediction. The prognosis predictive performance of the signature was good not only in the TCGA HCC cohort but also in the GSE14520 cohort. All these results indicated that the risk model developed from the five genes could be a useful indicator for HCC survival and to supplement the gap of the clinical prognostic signature of HCC.

The enrichment analysis yielded that the most significant enrichment term was the cell cycle and mitosis. CCNB2 and CDC20 participated in the mitotic cell cycle process. CCNB2, CDC20, NUSAP1, ASPM, and NCAPG involved in cell division. There are reports that CCNB2 is highly expressed in HCC (26). CCNB2 is involved in the development of HCC, which may be a prognostic factor (26). Regulatory protein encoded by CDC20 plays an important role in the occurrence and development of a variety of tumors, which may be related to its participation in the function of anaphase-promoting complex/cyclosome (APC/C) interaction in the cell cycle. Meanwhile, the increase in CDC20 expression has also been shown to be related to the occurrence and development of $\mathrm{HCC}(27,28)$. Because these evidences show that CCNB2 and CDC20 are directly related to the occurrence and development of HCC, we believe that they may

Peer) reviewing PDF | (2020:11:55265:1:1:NEW 11 Feb 2021) 
220

221

222

223

224

225

226

227

228

229

230

231

232

233

234

235

236

237

238

239

240

241

242

243

244

245

246

247

248

249

250

251

252

253

254

255

256

257

258

play the role of initiator and promoter in the process of HCC. NUSAP1 is one of the most critical microtubule and chromatin binding proteins, which can play a role during mitosis to crosslink microtubules. NUSAP1 expression levels in HCC tissues were higher than those in the adjacent tissues. With the high expression of NUSAP1 in HCC patients, the survival time of the patients also showed a significant decreasing trend (29). Unlike CCNB2 and CDC20, although NUSAP1 is highly expressed in HCC tissues with abnormal cell division, we have no direct evidence to prove its effect on the HCC process. Therefore, we believe that the highly expressed NUSAP1 may be temporarily used as a hepatocellular carcinoma Signs of disease. Increased expression of ASPM has been found in HCC. In addition, the expression level of ASPM has an important impact on the biological behavior of cancer cells or the prognosis of patients. ASPM overexpression is a molecular marker predicting poor prognosis (30). It has been reported that knocking out NCAPG can induce the division of HCC cells and even inhibit its deterioration in an in vitro environment. In contrast, the overexpression of NCAPG is also related to the recurrence of HCC patients (31). Studies have shown that the prognostic effect of NCAPG makes it a new biomarker for predicting whether recurrence will occur after surgical removal of the tumor (32). These evidences indicate that ASPM and NCAPG may be closely related to the prognosis of $\mathrm{HCC}$ patients, including positive, worsening or recurring prognostic results. Inhibiting the proliferation of HCC by targeted drugs to cause cancer cell apoptosis and curing cancer has become a new method of current cancer clinical treatment, such as Compound Kushen, which inhibits the cell cycle $(33,34)$. However, it not only provides new biomarkers for the target treatment of HCC, but also provides a new plan for the clinical management of HCC. In summary, consistent with our results, these 5 genes are all related to the prognosis of liver cancer. To our knowledge, the prognostic model associated with the five-gene signature may be a useful prognostic tool for liver cancer clinically. The risk score can be based on the mRNA expression levels of the five prognostic genes. In clinical practice, it may be more routine and cost-effective for all HCC patients. However, some limitations of our research should be considered. Firstly, we need to use more databases to verify the accuracy of this model. When we validated the five-gene signature by GSE14520 cohort, the median survival time of low-risk score group was undefined. It indicated that the follow-up time was not long enough in an original study. Secondly, at present, there are several reports that have screened out different genes signature, which needed to have a better and more accurate method to verify the effectiveness. Thirdly, expression profiling can only detect the change of gene expression level in our study, subsequent experiments are required for providing the information of those protein expression levels. Finally, we lack the molecular mechanisms of interaction between these genes, and we will incorporate these for further exploration.

\section{Conclusions}

This study aims to perform a comprehensive bioinformatics analysis of DEGs in four hepatocellular carcinoma datasets to discover potential biomarkers and predict their clinical effects. We established a five-gene signature (CCNB2, CDC20, NUSAP1, ASPM, and NCAPG)

Peer) reviewing PDF | (2020:11:55265:1:1:NEW 11 Feb 2021) 
259

260

261

262

263

264

265

266

267

268

269

270

271

272

273

274

275

276

277

278

279

280

281

282

283

284

285

286

287

288

289

290

291

292

293

294

295

296

297

to predict overall survival of HCC, which may contribute to the clinical decision-making of HCC treatment for different individuals.

\section{Acknowledgements}

The authors are grateful to all patients who provided samples to the public databases.

\section{References}

1. Bray F, Ferlay J, Soerjomataram I, Siegel R, Torre L, and Jemal A. 2018. Global cancer statistics 2018: GLOBOCAN estimates of incidence and mortality worldwide for 36 cancers in 185 countries. CA: a cancer journal for clinicians 68:394-424. 10.3322/caac. 21492

2. Siegel R, Miller K, and Jemal A. 2019. Cancer statistics, 2019. CA: a cancer journal for clinicians 69:7-34. 10.3322/caac.21551

3. Yan J, Zhou C, Guo K, Li Q, and Wang Z. 2019. A novel seven-lncRNA signature for prognosis prediction in hepatocellular carcinoma. Journal of cellular biochemistry 120:213223. 10.1002/jcb.27321

4. Mari A, Kimura S, Foerster B, Abufaraj M, D'Andrea D, Hassler M, Minervini A, Rouprêt M, Babjuk M, and Shariat S. 2019. A systematic review and meta-analysis of the impact of lymphovascular invasion in bladder cancer transurethral resection specimens. BJU international 123:11-21. 10.1111/bju.14417

5. Wan J, Liu H, Yang L, Ma L, Liu J, and Ming L. 2019. JMJD6 promotes hepatocellular carcinoma carcinogenesis by targeting CDK4. International journal of cancer 144:24892500. 10.1002/ijc.31816

6. Szuhai K, and Vermeer M. 2015. Microarray Techniques to Analyze Copy-Number Alterations in Genomic DNA: Array Comparative Genomic Hybridization and SingleNucleotide Polymorphism Array. The Journal of investigative dermatology 135:e37. 10.1038/jid.2015.308

7. Cao Y, Lu X, Li Y, Fu J, Li H, Li X, Chang Z, and Liu S. 2020. Identification of a six-gene metabolic signature predicting overall survival for patients with lung adenocarcinoma. PeerJ 8:e10320. 10.7717/peerj.10320

8. Wei H, Li J, Xie M, Lei R, and Hu B. 2018. Comprehensive analysis of metastasis-related genes reveals a gene signature predicting the survival of colon cancer patients. PeerJ 6:e5433. 10.7717/peerj.5433

9. Ashburner M, Ball C, Blake J, Botstein D, Butler H, Cherry J, Davis A, Dolinski K, Dwight S, Eppig J, Harris M, Hill D, Issel-Tarver L, Kasarskis A, Lewis S, Matese J, Richardson J, Ringwald M, Rubin G, and Sherlock G. 2000. Gene ontology: tool for the unification of biology. The Gene Ontology Consortium. Nature genetics 25:25-29. 10.1038/75556

10. Chin C, Chen S, Wu H, Ho C, Ko M, and Lin C. 2014. cytoHubba: identifying hub objects and sub-networks from complex interactome. BMC systems biology:S11. 10.1186/17520509-8-s4-s11

11. Consortium TGO. 2019. The Gene Ontology Resource: 20 years and still GOing strong. Nucleic acids research 47:D330-D338. 10.1093/nar/gky1055 
298

299

300

301

302

303

304

305

306

307

308

309

310

311

312

313

314

315

316

317

318

319

320

321

322

323

324

325

326

327

328

329

330

331

332

333

334

335

336

12. Kanehisa M, Furumichi M, Tanabe M, Sato Y, and Morishima K. 2017. KEGG: new perspectives on genomes, pathways, diseases and drugs. Nucleic acids research 45:D353D361. 10.1093/nar/gkw1092

13. Wang Z, Zhang G, Wu J, and Jia M. 2013. Adjuvant therapy for hepatocellular carcinoma: current situation and prospect. Drug discoveries \& therapeutics 7:137-143.

14. Subramanian A, Kuehn H, Gould J, Tamayo P, and Mesirov J. 2007. GSEA-P: a desktop application for Gene Set Enrichment Analysis. Bioinformatics (Oxford, England) 23:32513253. 10.1093/bioinformatics/btm369

15. Mardinoglu A, Gatto F, and Nielsen J. 2013. Genome-scale modeling of human metabolism - a systems biology approach. Biotechnology journal 8:985-996. 10.1002/biot.201200275

16. Rhodes D, Yu J, Shanker K, Deshpande N, Varambally R, Ghosh D, Barrette T, Pandey A, and Chinnaiyan A. 2004. ONCOMINE: a cancer microarray database and integrated datamining platform. Neoplasia (New York, NY) 6:1-6. 10.1016/s1476-5586(04)80047-2

17. Rhodes D, Kalyana-Sundaram S, Mahavisno V, Varambally R, Yu J, Briggs B, Barrette T, Anstet M, Kincead-Beal C, Kulkarni P, Varambally S, Ghosh D, and Chinnaiyan A. 2007. Oncomine 3.0: genes, pathways, and networks in a collection of 18,000 cancer gene expression profiles. Neoplasia (New York, NY) 9:166-180. 10.1593/neo.07112

18. Tang Z, Kang B, Li C, Chen T, and Zhang Z. 2019. GEPIA2: an enhanced web server for large-scale expression profiling and interactive analysis. Nucleic acids research 47:W556W560. 10.1093/nar/gkz430

19. Menyhárt O, Nagy Á, and Győrffy B. 2018. Determining consistent prognostic biomarkers of overall survival and vascular invasion in hepatocellular carcinoma. Royal Society open science 5:181006. 10.1098/rsos.181006

20. Huitzil-Melendez F, Capanu M, O'Reilly E, Duffy A, Gansukh B, Saltz L, and Abou-Alfa G. 2010. Advanced hepatocellular carcinoma: which staging systems best predict prognosis? Journal of clinical oncology : official journal of the American Society of Clinical Oncology 28:2889-2895. 10.1200/jco.2009.25.9895

21. Cao Y, Lu X, Li Y, Fu J, Li H, Li X, Chang Z, and Liu S. 2020. Identification of a six-gene metabolic signature predicting overall survival for patients with lung adenocarcinoma. PeerJ 8:e10320. 10.7717/peerj.10320

22. Long J, Zhang L, Wan X, Lin J, Bai Y, Xu W, Xiong J, and Zhao H. 2018. A four-genebased prognostic model predicts overall survival in patients with hepatocellular carcinoma. Journal of cellular and molecular medicine 22:5928-5938. 10.1111/jcmm.13863

23. Liu S, Miao C, Liu J, Wang C, and Lu X. 2018. Four differentially methylated gene pairs to predict the prognosis for early stage hepatocellular carcinoma patients. Journal of cellular physiology 233:6583-6590. 10.1002/jcp.26256

24. Wang Z, Teng D, Li Y, Hu Z, Liu L, and Zheng H. 2018. A six-gene-based prognostic signature for hepatocellular carcinoma overall survival prediction. Life sciences 203:83-91. 10.1016/j.1fs.2018.04.025

Peer) reviewing PDF | (2020:11:55265:1:1:NEW 11 Feb 2021) 
337

338

339

340

341

342

343

344

345

346

347

348

349

350

351

352

353

354

355

356

357

358

359

360

361

362

363

364

365

366

367

368

369

370

371

372

373

374

25. Kong J, Wang T, Zhang Z, Yang X, Shen S, and Wang W. 2019. Five Core Genes Related to the Progression and Prognosis of Hepatocellular Carcinoma Identified by Analysis of a Coexpression Network. DNA and cell biology 38:1564-1576. 10.1089/dna.2019.4932

26. Li R, Jiang X, Zhang Y, Wang S, Chen X, Yu X, Ma J, and Huang X. 2019. Cyclin B2 Overexpression in Human Hepatocellular Carcinoma is Associated with Poor Prognosis. Archives of medical research 50:10-17. 10.1016/j.arcmed.2019.03.003

27. Li J, Gao J, Du J, Huang Z, and Wei L. 2014. Increased CDC20 expression is associated with development and progression of hepatocellular carcinoma. International journal of oncology 45:1547-1555. 10.3892/ijo.2014.2559

28. Liu M, Zhang Y, Liao Y, Chen Y, Pan Y, Tian H, Zhan Y, and Liu D. 2015. Evaluation of the Antitumor Efficacy of RNAi-Mediated Inhibition of CDC20 and Heparanase in an Orthotopic Liver Tumor Model. Cancer biotherapy \& radiopharmaceuticals 30:233-239. $10.1089 / \mathrm{cbr} .2014 .1799$

29. Wang Y, Ju L, Xiao F, Liu H, Luo X, Chen L, Lu Z, and Bian Z. 2019. Downregulation of nucleolar and spindle-associated protein 1 expression suppresses liver cancer cell function. Experimental and therapeutic medicine 17:2969-2978. 10.3892/etm.2019.7314

30. Lin S, Pan H, Liu S, Jeng Y, Hu F, Peng S, Lai P, and Hsu H. 2008. ASPM is a novel marker for vascular invasion, early recurrence, and poor prognosis of hepatocellular carcinoma. Clinical cancer research : an official journal of the American Association for Cancer Research 14:4814-4820. 10.1158/1078-0432.Ccr-07-5262

31. Zhang Q, Su R, Shan C, Gao C, and Wu P. 2018. Non-SMC Condensin I Complex, Subunit G (NCAPG) is a Novel Mitotic Gene Required for Hepatocellular Cancer Cell Proliferation and Migration. Oncology research 26:269-276. 10.3727/096504017x15075967560980

32. Wang Y, Gao B, Tan P, Handoko Y, Sekar K, Deivasigamani A, Seshachalam V, OuYang H, Shi M, Xie C, Goh B, Ooi L, and Man Hui K. 2019. Genome-wide CRISPR knockout screens identify NCAPG as an essential oncogene for hepatocellular carcinoma tumor growth. FASEB journal : official publication of the Federation of American Societies for Experimental Biology 33:8759-8770. 10.1096/fj.201802213RR

33. Feitelson M, Arzumanyan A, Kulathinal R, Blain S, Holcombe R, Mahajna J, Marino M, Martinez-Chantar M, Nawroth R, Sanchez-Garcia I, Sharma D, Saxena N, Singh N, Vlachostergios P, Guo S, Honoki K, Fujii H, Georgakilas A, Bilsland A, Amedei A, Niccolai E, Amin A, Ashraf S, Boosani C, Guha G, Ciriolo M, Aquilano K, Chen S, Mohammed S, Azmi A, Bhakta D, Halicka D, Keith W, and Nowsheen S. 2015. Sustained proliferation in cancer: Mechanisms and novel therapeutic targets. Seminars in cancer biology:S25-S54. 10.1016/j.semcancer.2015.02.006

34. Cui J, Qu Z, Harata-Lee Y, Nwe Aung T, Shen H, Wang W, and Adelson D. 2019. Cell cycle, energy metabolism and DNA repair pathways in cancer cells are suppressed by Compound Kushen Injection. BMC cancer 19:103. 10.1186/s12885-018-5230-8

Peer) reviewing PDF | (2020:11:55265:1:1:NEW 11 Feb 2021) 
Figure 1

The flow chart showing our protocol for studying the mRNA prognostic characteristics of HCC.

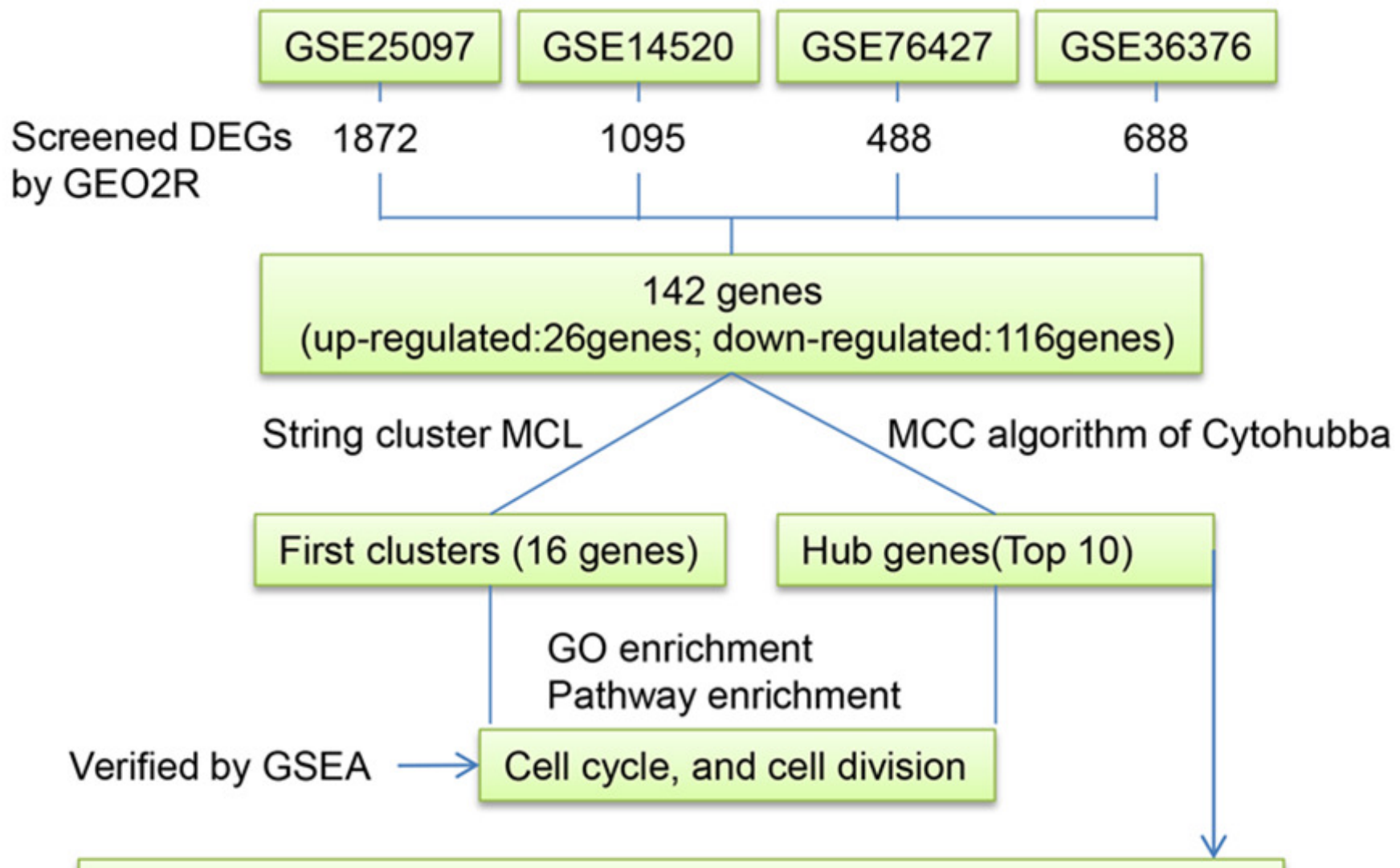

Expression in liver cancer and Prognostic analysis of top 10 genes

Pearson correlation test

Independence of 5 genes prognostic model

$\downarrow$

Verified by Overall Survival analysis 
Figure 2

Screening of Commonly Differentially Expressed Genes in the four hepatocellular carcinoma datasets.

(A) DEGs in GSE14520 are displayed in the volcano plot. (B) DEGs in GSE25097. (C) DEGs in GSE36376. (D) DEGs in GSE76427. Statistically significant DEGs were defined with adjusted $\mathrm{P}<0.05$ and $|\log \mathrm{FC}|>1$ as the threshold value. (E) All the DEGs common to the four datasets are displayed in the Venn diagrams. (F) Up-regulated DEGs common to the four datasets. (G) Down-regulated DEGs common to the four datasets.
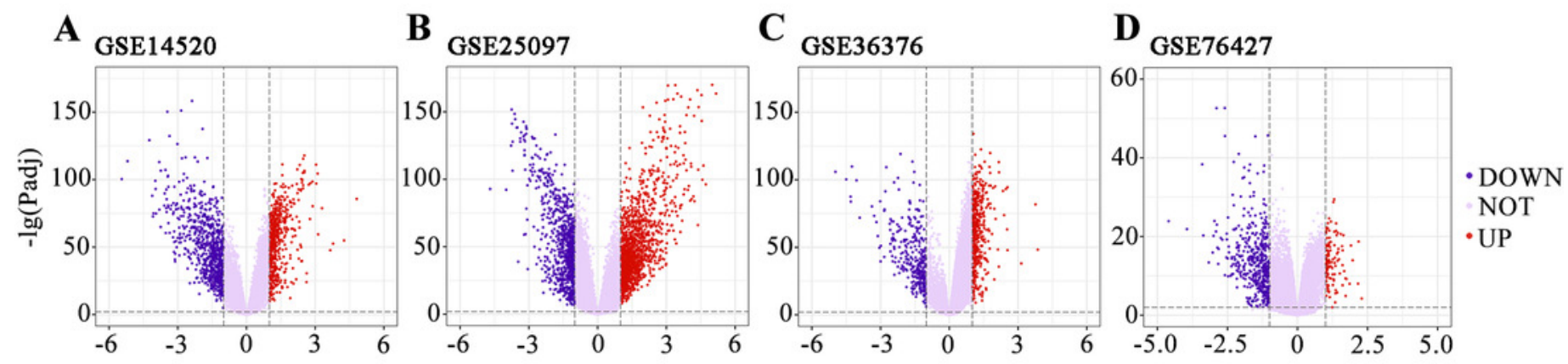

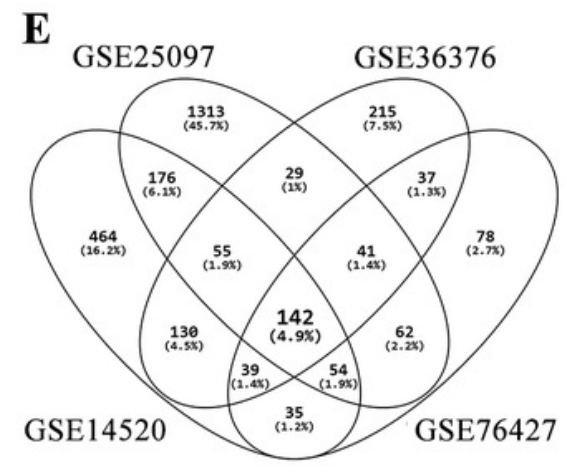

All genes

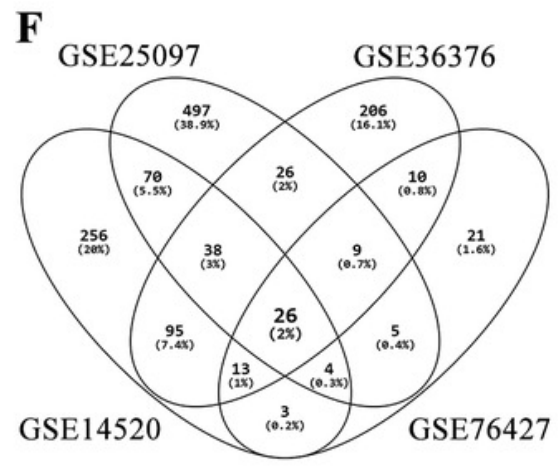

Up-regulated genes

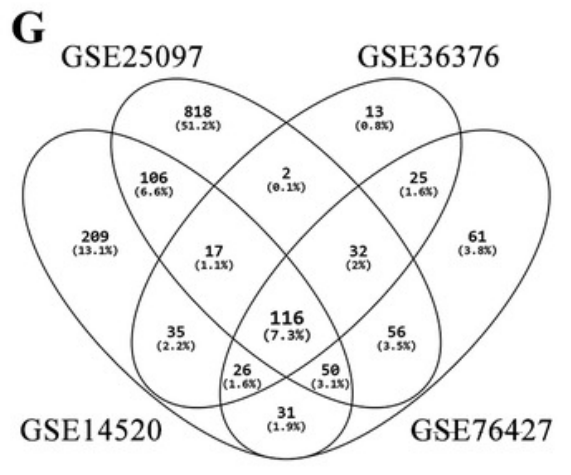

Down-regulated genes 
Figure 3

Prediction and identification of hub genes in HCC.

(A) A total of 142 DEGs were filtered into the PPI interaction network using the STRING online tools. Red nodes were first cluster which was created using the MCL clustering algorithm. (B) The top-10 hub genes ranked by the MCC algorithm of Cytohubba.

A

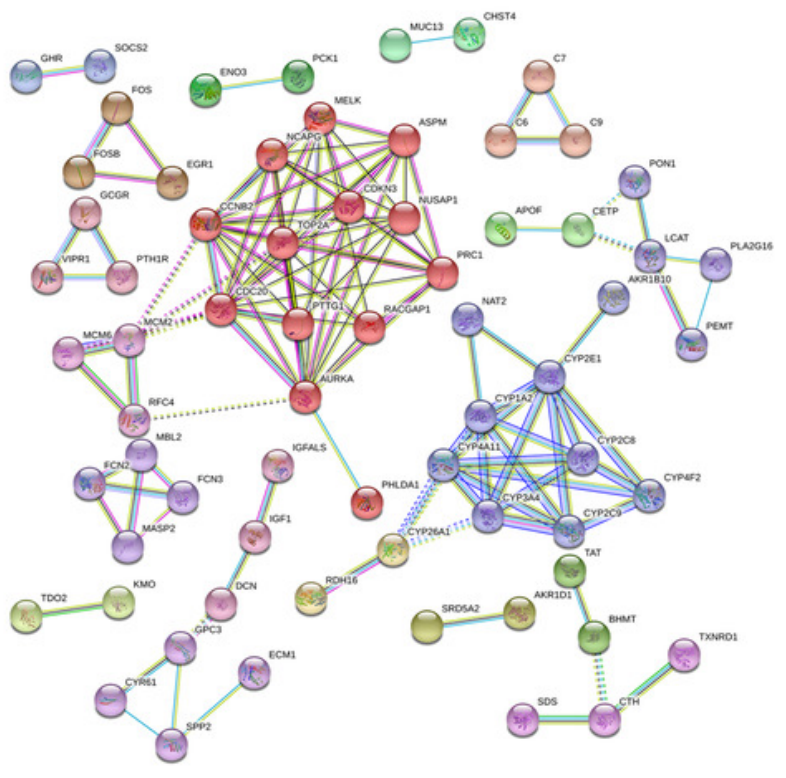

B

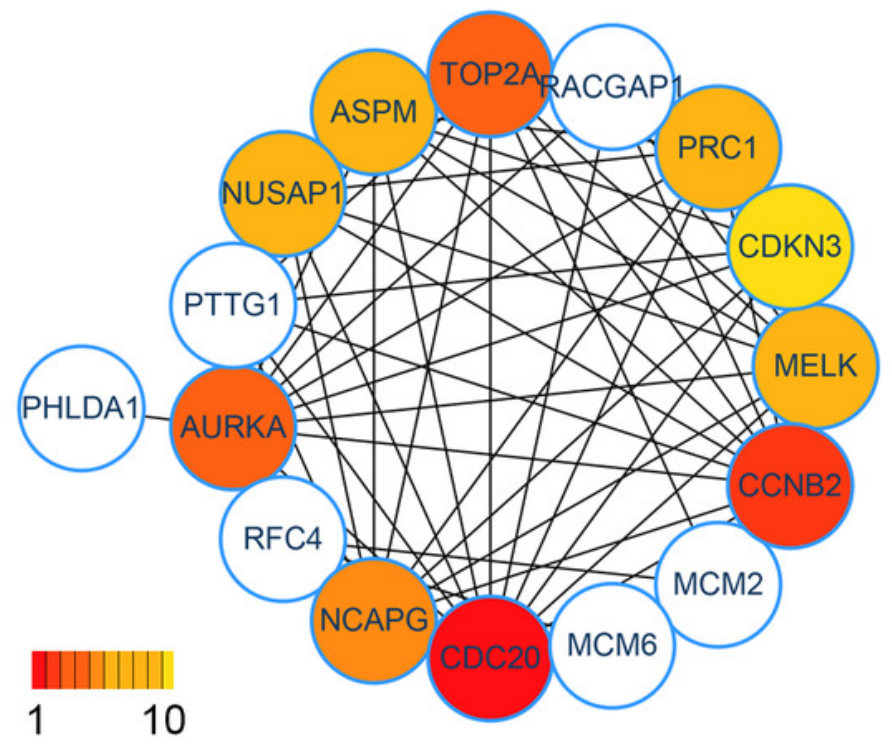




\section{Figure 4}

Gene Ontology (GO) and Kyoto Encyclopedia of Genes and Genomes (KEGG) analysis of the hub genes.

(A) Significant enrichment of GO annotation and KEGG pathway of hub genes in hepatocellular carcinoma by Metascape $(P<0.05)$. (B) Network of enriched terms: colored by cluster ID, where nodes that share the same cluster ID are typically close to each other. (C) Network of enriched terms: colored by $p$-value, where terms containing more genes tend to have a more significant p-value. (D) Protein-protein interaction network and Molecular Complex Detection (MCODE) components identified in the gene lists. (E) Visualization of GSEA results for cell cycles in GSE14520. (F) Visualization of GSEA results for cell cycles in GSE25097. (G) Visualization of GSEA results for cell cycles in GSE36376. (H) Visualization of GSEA results for cell cycles in GSE76427. NES, normalized enrichment score; FDR, adjusted p value. 
$\mathbf{A}$

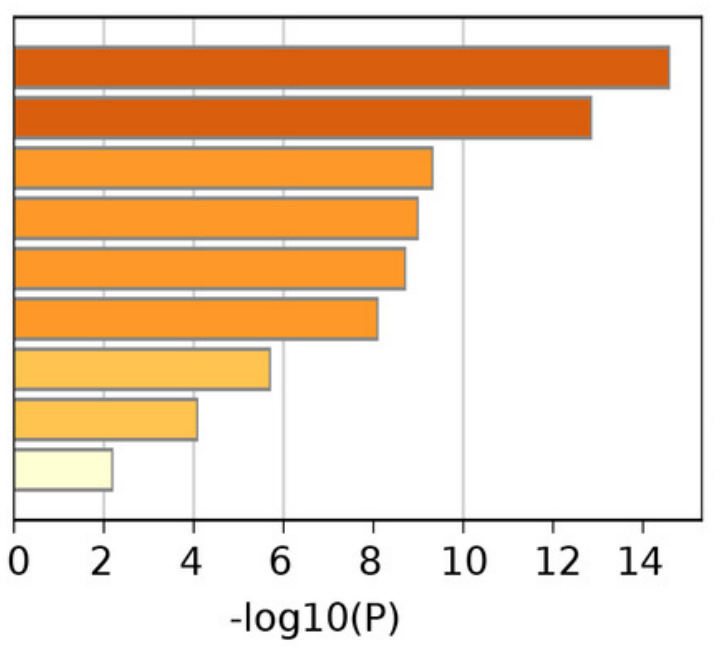

R-HSA-69278: Cell Cycle, Mitotic

GO:0051301: cell division

GO:0044772: mitotic cell cycle phase transition

GO:0071103: DNA conformation change

ko04110: Cell cycle

GO:0008094: DNA-dependent ATPase activity

GO:0000922: spindle pole

GO:0061351: neural precursor cell proliferation

GO:0043065: positive regulation of apoptotic process

B

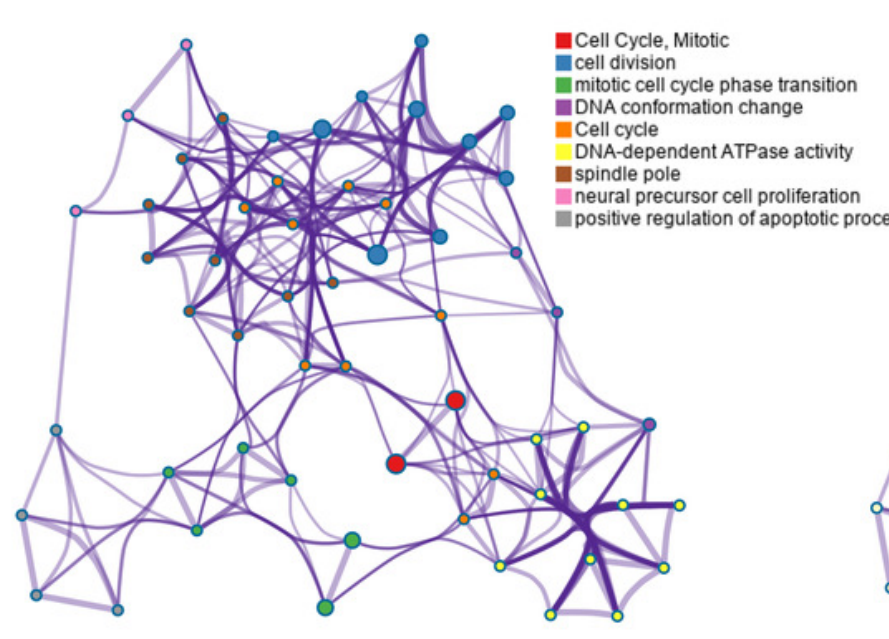

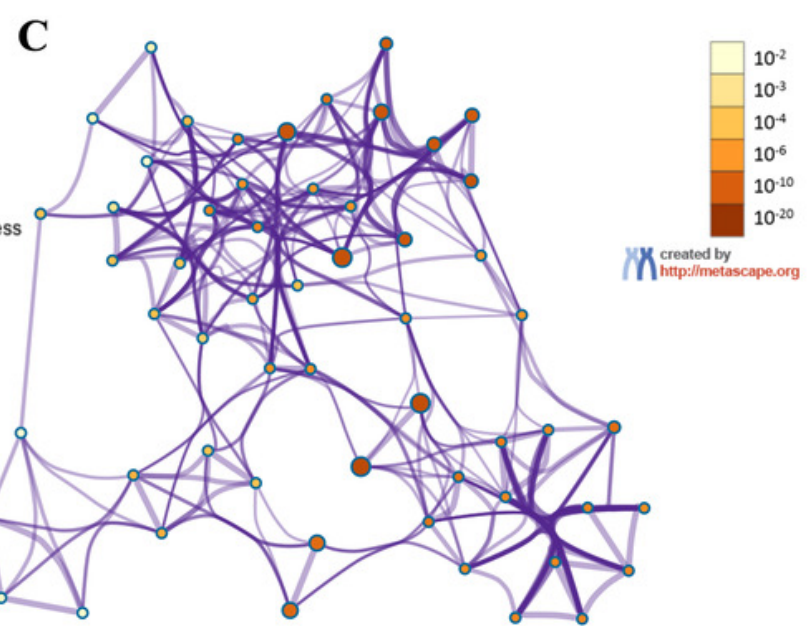

D

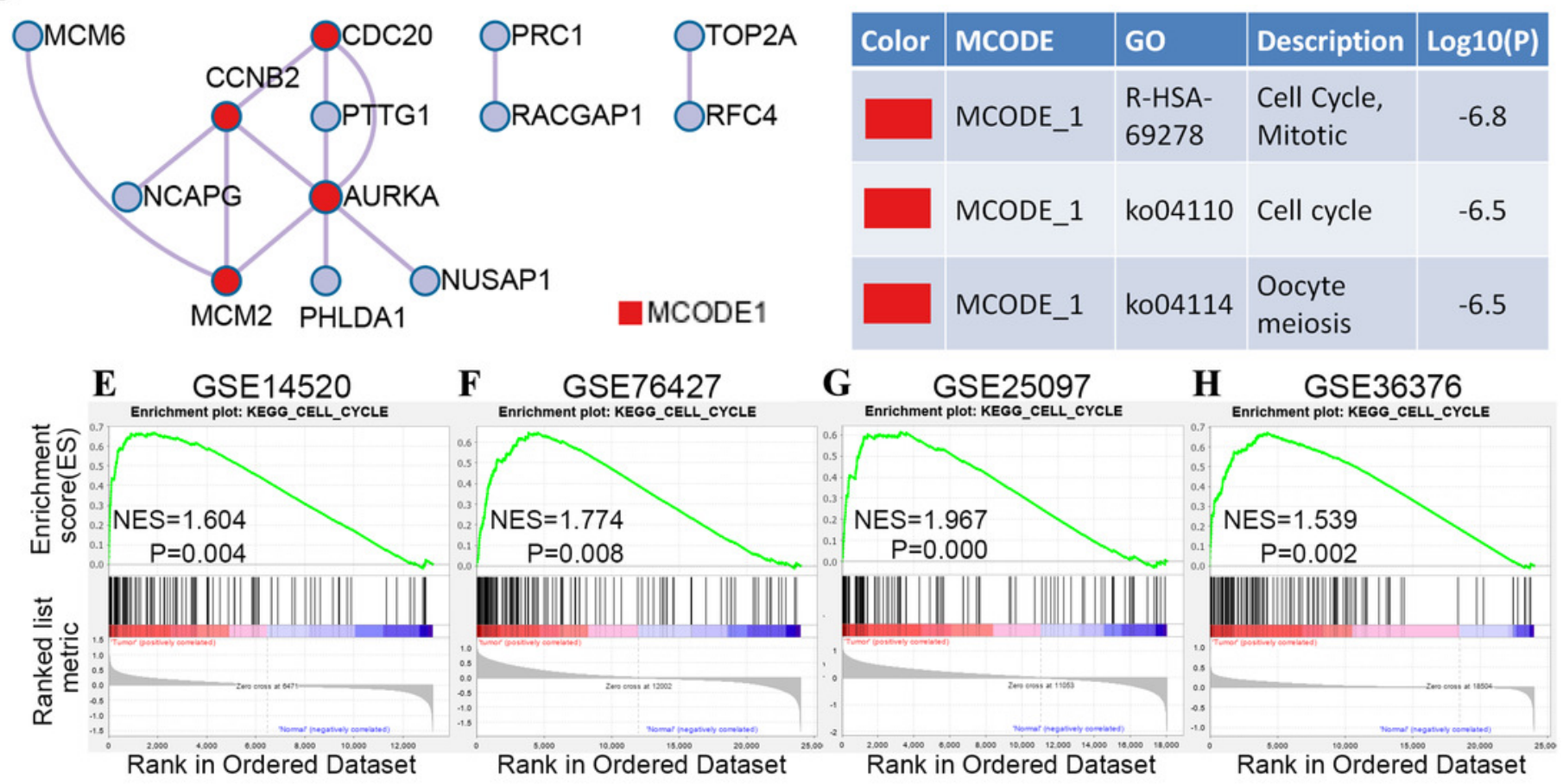




\section{Figure 5}

Differential analysis of transcription levels of hub genes in hepatocellular carcinoma.

(A) The transcription levels of hub genes in different types of cancers. The graphs showed the numbers of datasets with statistically significant mRNA over-expression (red) or downexpression (blue) of these genes. Differences in the level of transcription of hub genes are displayed in the box plot (GEPIA) which derived from gene expression data for GEPIA comparing the expression of hub genes in HCC tissue $(n=369$, pink) and normal tissues ( $n=160$, gray). Including (B) CDC20, (C) CCNB2, (D) AURKA, (E) NCAPG, (F) ASPM, (G) TOP2A, (H) NUSAP1, (I) PRC1, (J) MELK and (K) CDKN3. * show that $p$ value is less than 0.01. 


\begin{tabular}{|c|c|c|c|c|c|c|c|c|c|c|c|c|c|c|c|c|c|c|c|c|}
\hline \multirow{2}{*}{$\begin{array}{r}\text { Analysis Type by Cancer } \\
\text { Bladder Cancer }\end{array}$} & \multicolumn{2}{|c|}{$\begin{array}{l}\text { Cancer } \\
\text { vs. } \\
\text { Normal } \\
\text { CDC20 }\end{array}$} & \multicolumn{2}{|c|}{$\begin{array}{l}\text { Cancer } \\
\text { vs. } \\
\text { Normal } \\
\text { CCNB2 }\end{array}$} & \multicolumn{2}{|c|}{$\begin{array}{l}\text { Cancer } \\
\text { vs. } \\
\text { Normal } \\
\text { AURKA }\end{array}$} & \multicolumn{2}{|c|}{$\begin{array}{l}\text { Cancer } \\
\text { vs. } \\
\text { Normal } \\
\text { NCAPG }\end{array}$} & \multicolumn{2}{|c|}{$\begin{array}{l}\text { Cancer } \\
\text { vs. } \\
\text { Normal } \\
\text { ASPM }\end{array}$} & \multicolumn{2}{|c|}{$\begin{array}{c}\text { Cancer } \\
\text { vs. } \\
\text { Normal } \\
\text { TOP2A }\end{array}$} & \multicolumn{2}{|c|}{$\begin{array}{c}\text { Cancer } \\
\text { vs. } \\
\text { Normal } \\
\text { NUSAP1 }\end{array}$} & \multicolumn{2}{|c|}{$\begin{array}{l}\text { Cancer } \\
\text { vs. } \\
\text { Normal } \\
\text { PRC1 }\end{array}$} & \multicolumn{2}{|c|}{$\begin{array}{l}\text { Cancer } \\
\text { vs. } \\
\text { Normal } \\
\text { MELK }\end{array}$} & \multicolumn{2}{|c|}{$\begin{array}{c}\text { Cancer } \\
\text { vs. } \\
\text { Normal } \\
\text { CDKN3 }\end{array}$} \\
\hline & 6 & & 4 & & 5 & & 4 & & 1 & & 8 & & 6 & & 5 & & 4 & & 2 & \\
\hline \multirow{2}{*}{$\begin{array}{r}\text { Brain and CNS Cancer } \\
\text { Breast Cancer }\end{array}$} & 6 & 1 & 7 & 1 & 3 & 1 & 5 & 1 & 4 & & 13 & 1 & 8 & 1 & 4 & 1 & 9 & 1 & 3 & 1 \\
\hline & 16 & 1 & 24 & & 20 & 1 & 19 & 1 & 18 & 1 & 29 & 1 & 23 & 1 & 20 & & 23 & 1 & 22 & 1 \\
\hline \multirow{2}{*}{$\begin{array}{r}\text { Cervical Cancer } \\
\text { Colorectal Cancer }\end{array}$} & 3 & & 3 & & 3 & & 4 & & 2 & & 4 & & 4 & & 4 & & 4 & & 4 & \\
\hline & 9 & & 7 & & 16 & & 15 & & 10 & & 21 & & 16 & & 9 & & 18 & & 10 & \\
\hline \multirow{2}{*}{$\begin{array}{r}\text { Esophageal Cancer } \\
\text { Gastric Cancer }\end{array}$} & 2 & & 3 & & 4 & & 1 & & & & 2 & & 3 & & 3 & & 4 & & 3 & \\
\hline & 3 & & 3 & & 5 & & 4 & & 7 & & 10 & & 5 & & 9 & & 4 & & 6 & \\
\hline \multirow{3}{*}{$\begin{array}{r}\text { Head and Neck Cancer } \\
\text { Kidney Cancer } \\
\text { Leukemia }\end{array}$} & 8 & & 6 & & 11 & & 6 & & 1 & & 11 & & 3 & & 5 & & 8 & & 7 & \\
\hline & & & 2 & & 1 & 2 & 1 & & & & 4 & & 4 & & 4 & & 1 & & & \\
\hline & 1 & 8 & 2 & 8 & 1 & 8 & & 3 & & 4 & 2 & 9 & 3 & 3 & 3 & 5 & 3 & 6 & & 9 \\
\hline Liver Cancer & 3 & & 3 & & 4 & & 3 & & 1 & & 4 & & 4 & & 3 & & 3 & & 4 & \\
\hline \multirow{2}{*}{$\begin{array}{r}\text { Lung Cancer } \\
\text { Lymphoma }\end{array}$} & 12 & 1 & 11 & & 14 & & 5 & & 4 & & 21 & 1 & 11 & & 7 & & 13 & & 15 & 1 \\
\hline & 11 & 2 & 6 & 3 & 8 & 1 & 3 & & 7 & 1 & 11 & 1 & 6 & 1 & 5 & & 9 & & 12 & 1 \\
\hline \multirow{2}{*}{$\begin{array}{r}\text { Melanoma } \\
\text { Myeloma }\end{array}$} & 2 & & 1 & & 2 & & 2 & & & & 3 & 1 & 3 & & 1 & & 1 & & 2 & \\
\hline & & 1 & & & & & 2 & & & & & & & & 3 & & & & & \\
\hline \multirow{2}{*}{$\begin{array}{r}\text { Other Cancer } \\
\text { Ovarian Cancer }\end{array}$} & 5 & 2 & 7 & 7 & 3 & 1 & 5 & 1 & 1 & & 10 & 5 & 8 & & 6 & 6 & 7 & 1 & 4 & 9 \\
\hline & 4 & & 4 & & 3 & & 2 & & 1 & & 7 & & 3 & & 6 & & 2 & & 4 & \\
\hline \multirow{2}{*}{$\begin{array}{r}\text { Pancreatic Cancer } \\
\text { Prostate Cancer }\end{array}$} & 2 & & 1 & & 2 & 1 & 2 & & & & 6 & & 4 & & 3 & & 3 & & 3 & \\
\hline & & & 1 & & 1 & & 1 & & 1 & & 2 & & 2 & & & & & & 1 & \\
\hline Sarcoma & 12 & 1 & 11 & 1 & 10 & 1 & 9 & & & & 12 & & 11 & 1 & 12 & & 11 & & 8 & \\
\hline \multirow{2}{*}{\begin{tabular}{|r|} 
Significant Unique Analyses \\
Total Unique Analyses
\end{tabular}} & 103 & 17 & 104 & 20 & 115 & 16 & 92 & 6 & 58 & 6 & 178 & 19 & 125 & 7 & 109 & 12 & 126 & 9 & 109 & 22 \\
\hline & 41 & & 41 & & 43 & & 2 & & 1 & & 36 & & 29 & & 29 & & 33 & & 44 & \\
\hline
\end{tabular}

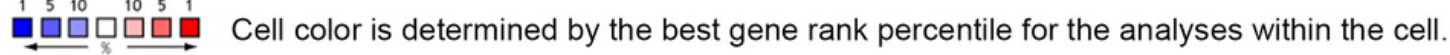
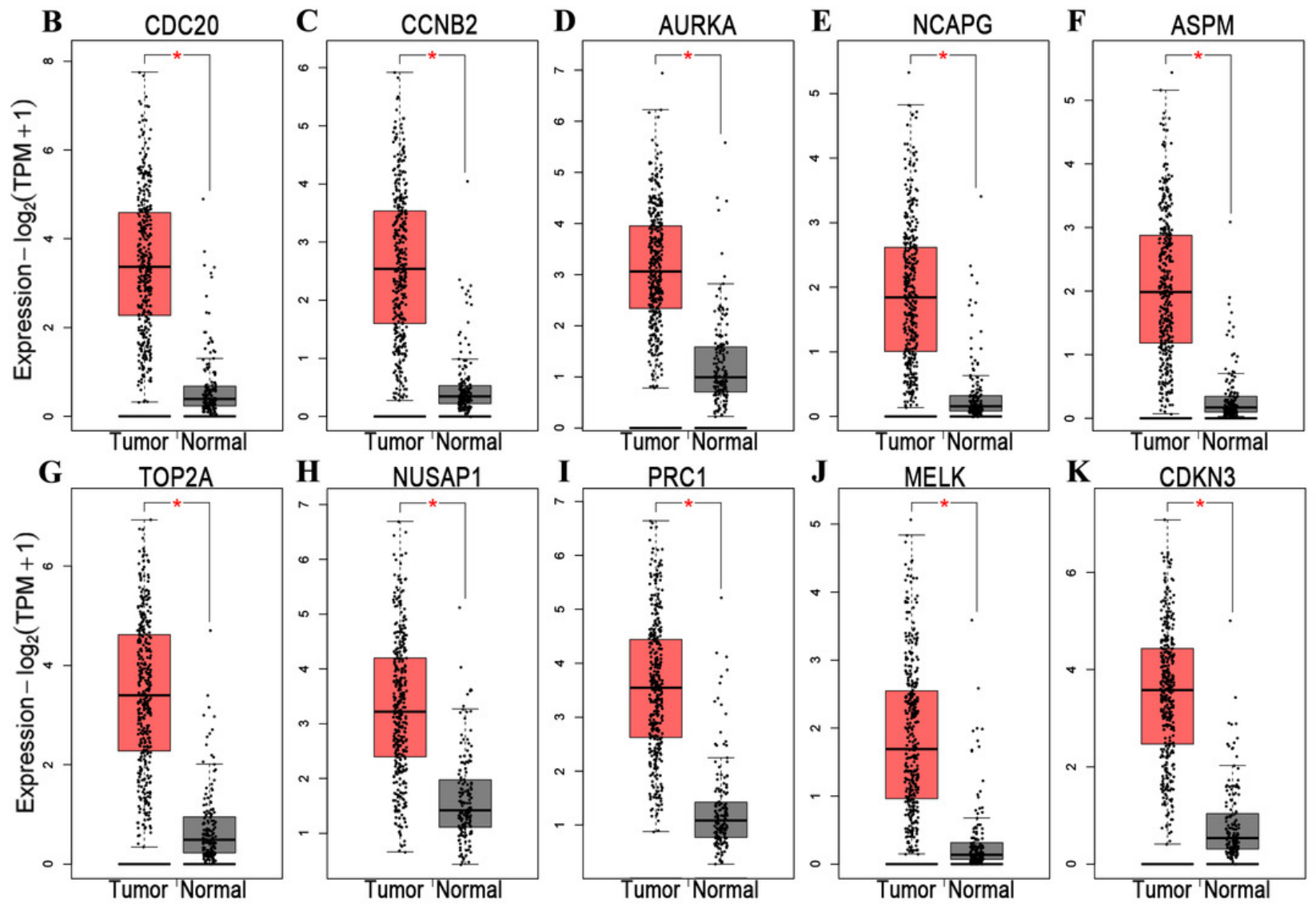

K

CDKN3

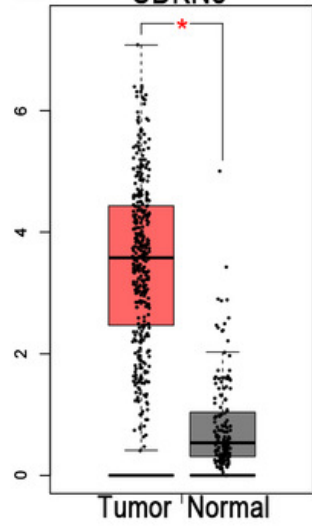




\section{Figure 6}

Kaplan-Meier analysis for ten hub genes in HCC.

Figure 6 is divided into three parts. First, overall survival (OS) curves of ten hub genes, including (A) CDC20, (B) CCNB2, (C) AURKA, (D) NCAPG, (E) ASPM, (F) TOP2A, (G) NUSAP1, (H) PRC1, (I) MELK and (J) CDKN3. Second, Progression-free survival (PFS) curves of (K) CDC20, (L) CCNB2, (M) AURKA, (N) NCAPG, (O) ASPM, (P) TOP2A, (Q) NUSAP1, (R) PRC1, (S) MELK and (T) CDKN3. Third, Relapse-free survival (RFS) curves of (U) CDC20, (V) CCNB2, (W) AURKA, (X) NCAPG, (Y) ASPM, (Z) TOP2A, (AA) NUSAP1, (BB) PRC1, (CC) MELK and (DD) CDKN3. 

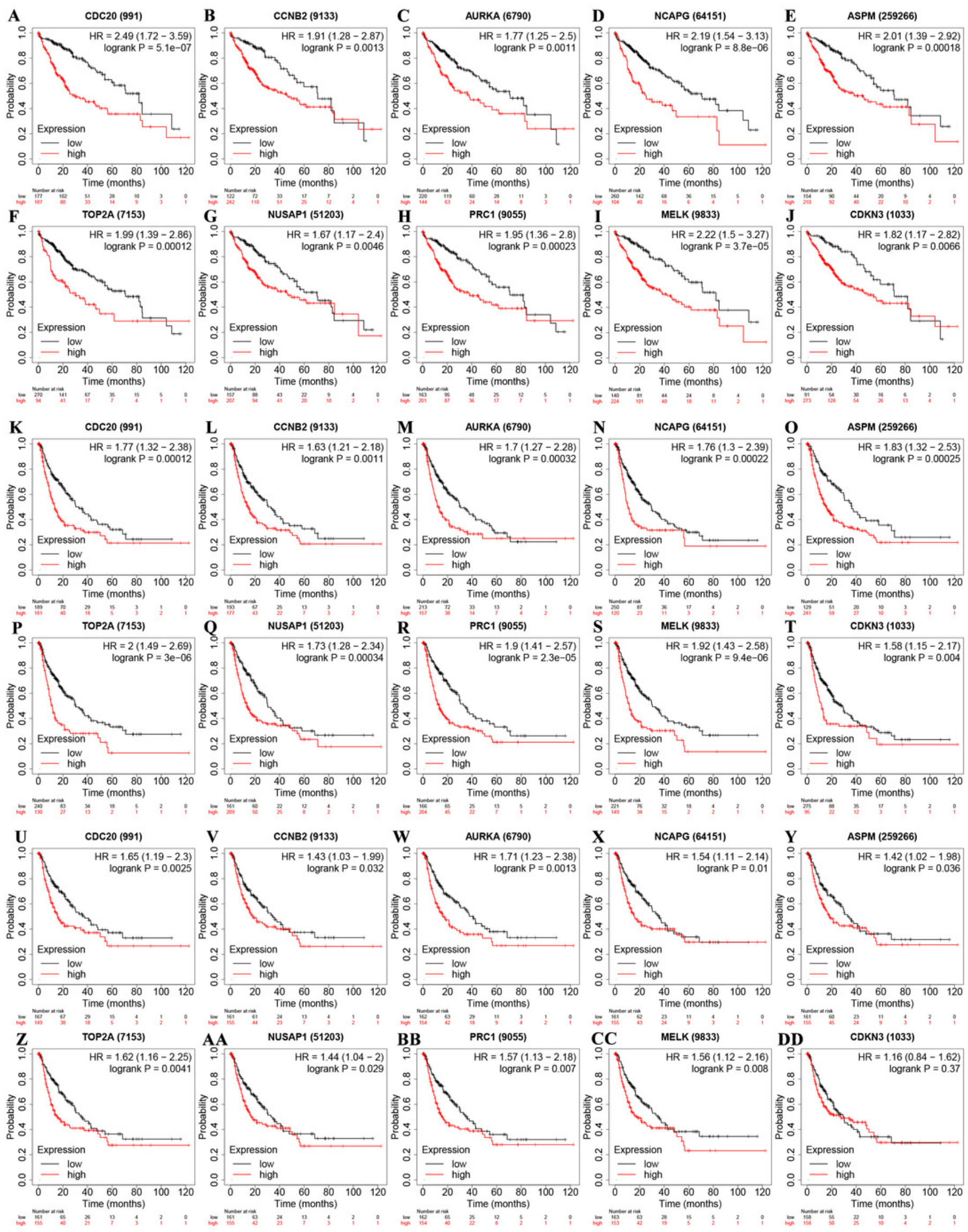


\section{Figure 7}

Kaplan-Meier analysis predicting overall survival for patients with HCC.

(A) Overall survival (OS) curves of ten hub genes in TCGA-LIHC cohort. (B) Overall survival (OS) curves of five-gene signature in TCGA-LIHC cohort. (C) The expression alteration profiles of the six genes in the TCGA-LIHC cohort. (D) Overall survival (OS) curves of five-gene signature compared the survival difference between the high-score and low-score group in TCGA-LIHC cohort. (E) Overall survival (OS) curves of five-gene signature compared the difference between the high-score and low-score group in GSE14520 cohort. 


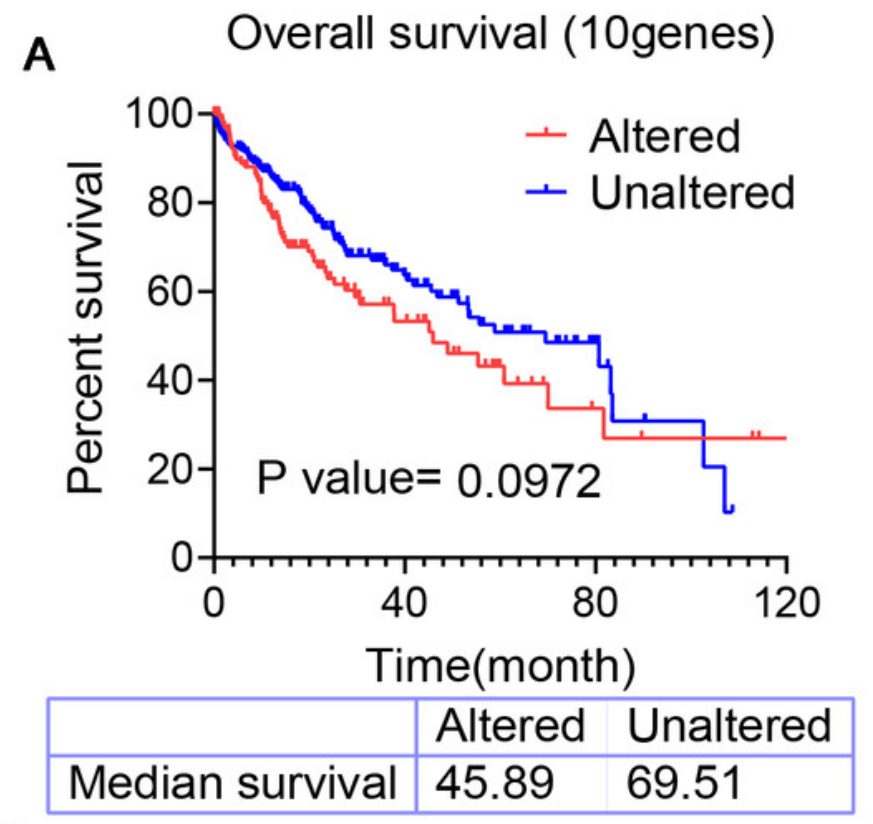

B Overall survival (5 genes)

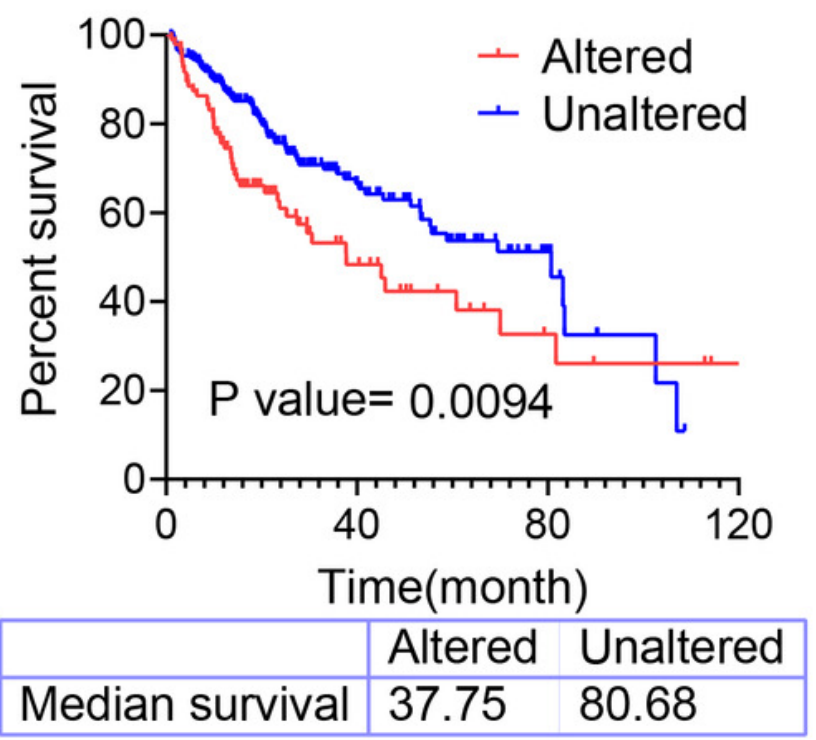

C

Survival Status

CDC20 6\%

CCNB2 $\quad 9 \%$

NUSAP1 $\quad 8 \%$

NCAPG $\quad 6 \%$

ASPM $\quad 16 \%$

Genetic Alteration || Missense Mutation (unknown significance) || Truncating Mutation (unknown significance) | Amplification | Deep Deletion | mRNA High Survival Status I0:LIVING I1:DECEASED

5 genes are altered in $93(25 \%)$ of queried samples

D

Overall survival (TCGA)

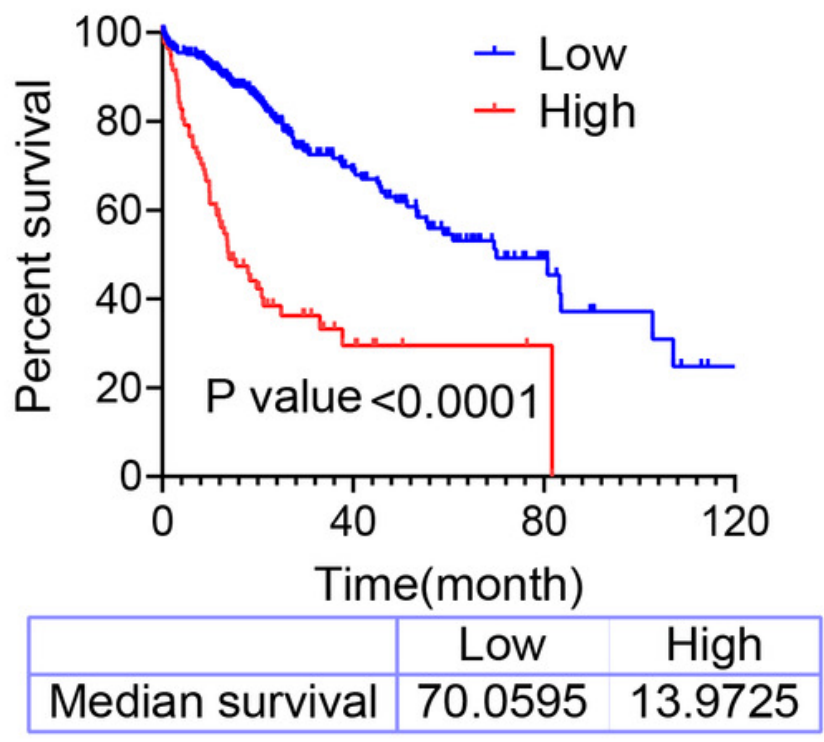

E

Overall survival (GSE14520)

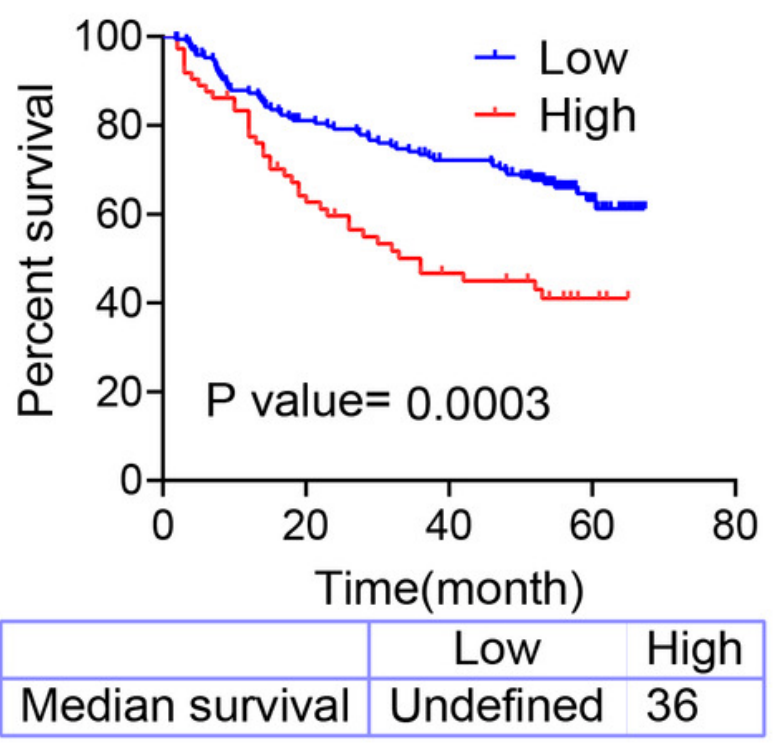


Table $\mathbf{1}$ (on next page)

Basic information of four GEO datasets. 
1 Table 1. Basic information of four GEO datasets.

\begin{tabular}{cccc}
\hline GEO datasets & platform & hepatocellular carcinoma samples & Non-tumor samples \\
\hline GSE25097 & GPL16087 & 243 & 268 \\
GSE47197 & GPL16699 & 61 & 63 \\
GSE54236 & GPL6480 & 80 & 81 \\
GSE60502 & GPL96 & 18 & 18 \\
\hline
\end{tabular}

2 
Table 2 (on next page)

Kaplan-Meier analysis for ten hub genes in HCC 
1 Table 2. Kaplan-Meier analysis for ten hub genes in HCC

\begin{tabular}{cccccccccc}
\hline \multirow{2}{*}{ Gene Symbol } & \multicolumn{3}{c}{ OS(months) } & \multicolumn{3}{c}{ RFS(months) } & \multicolumn{3}{c}{ PFS(months) } \\
\cline { 2 - 10 } & Low & High & Logrank P & Low & High & Logrank P & Low & High & Logrank P \\
\hline CDC20 & 81.9 & 30 & $5.1 \mathrm{e}-7$ & 36.1 & 13.27 & 0.0006 & 33 & 13.27 & 0.0001 \\
CCNB2 & 71 & 46.6 & 0.0013 & 36.1 & 16.73 & 0.0069 & 33 & 13.83 & 0.0011 \\
AURKA & 71 & 37.8 & 0.0011 & 40.97 & 15.63 & 0.0002 & 30.4 & 12.87 & 0.0003 \\
NCAPG & 70.5 & 25.2 & $8.8 \mathrm{e}-6$ & 34.4 & 11.67 & 0.0006 & 29.73 & 10.4 & 0.0002 \\
ASPM & 71 & 45.7 & 0.0002 & 33 & 13.27 & 0.0031 & 36.27 & 15.83 & 0.0002 \\
TOP2A & 71 & 30 & 0.0001 & 36.1 & 11.83 & 0.0001 & 30.4 & 11.33 & $3.0 \mathrm{e}-6$ \\
NUSAP1 & 70.5 & 46.6 & 0.0046 & 36.1 & 13.27 & 0.0010 & 30.4 & 13.33 & 0.0003 \\
PRC1 & 71 & 38.3 & 0.0002 & 36.1 & 12.87 & 0.0005 & 34.4 & 13.27 & $2.3 \mathrm{e}-5$ \\
MELK & 81.9 & 42.4 & $3.7 \mathrm{e}-5$ & 37.23 & 12.87 & $2.5 \mathrm{e}-5$ & 30.4 & 11.6 & $9.4 \mathrm{e}-6$ \\
CDKN3 & 71 & 49.7 & 0.0066 & 30.4 & 17.9 & 0.0219 & 27.6 & 11.83 & 0.004 \\
\hline
\end{tabular}

2

3

4 